\title{
The Effect of Knee Joint Traction Therapy on Pain, Physical Function, and Depression in Patients with Degenerative Arthritis
}

\author{
Min Sun Choi', Dong Kyu Lee ${ }^{2}$ \\ 'Department of Orthopedic Surgery, Sunhan Hospital, Gwangju; ²Department of Physical Therapy, Sunhan Hospital, Gwangju Korea
}

Purpose: To identify the effect of knee joint traction therapy on pain, physical function, and depression in patients with degenerative arthritis.

Methods: In total, 30 patients with degenerative arthritis were randomly assigned to one of two groups: the experimental group, who underwent knee joint traction therapy, and the control group, who underwent general physical therapy (15 patients per group). Pain was measured using the visual analogue scale (VAS), physical function was measured using the Western ontario and McMaster universities osteoarthritis (WOMAC) index, and depression was measured using the Beck depression inventory (BDI). The VAS, WOMAC score, and BDI score were recorded before and after the 4-week treatment.

Results: As a result of comparison within groups, the experimental and control group showed significant difference for VAS, WOMAC and $\mathrm{BDI}$ after the experiment $(\mathrm{p}<0.05)$. In comparison between the two groups, the experimental group in which knee joint traction was applied showed more significant change in VAS, WOMAC and BDI than the control group $(p<0.05)$.

Conclusion: This study showed that knee joint traction therapy was effective in improving pain, physical function, and depression in patients with degenerative arthritis.

Keywords: Knee joint traction, VAS, WOMAC, BDI

\section{INTRODUCTION}

Degenerative arthritis is one of the most common diseases worldwide. ${ }^{1}$ This condition is a progressive disease that causes histological changes such as cartilage degeneration and proliferation of bone, cartilage, and surrounding connective tissue, which results in a severe functional limitations in patients. ${ }^{2,3}$ Knee joint degenerative arthritis is a disease that causes pain, functional limitation, and disability, and its incidence is gradually rising, especially in the elderly population. ${ }^{4.5}$ One of the main symptoms observed among patients with knee joint degenerative arthritis is pain, ${ }^{6}$ and a high correlation has been found between pain and limited physical function during activities that use the knee in patients with degenerative arthritis., ${ }^{4,6}$ Degenerative arthritis is a disease that can cause physical problems such as functional disorders and pain, as well as decreased quality of life, which causes psychosocial problems.? The persistent pain and

Received Sep 16, 2019 Revised Oct 11, 2019

Accepted Oct 14, 2019

Corresponding author Dong Kyu Lee

E-mail Idkpt@hanmail.net functional disorders also lead to sociopsychological issues, such as acute depression and declined quality of life. In general, the goals of the clinical management of knee degenerative arthritis are to provide pain relief and to maintain or to improve functionality. ${ }^{2,6} \mathrm{~A}$ diverse range of surgical and non-surgical options are available for treating degenerative arthritis, yet each therapeutic modality has its respective limitations and side effects. ${ }^{8}$ Furthermore, no treatments have been developed to completely cure knee degenerative arthritis. Therefore, the goal of clinical treatments is to relieve pain, maintain or improve joint function, and reduce joint stiffening or deformation. ${ }^{9}$

Since there are difficulties in the current treatment of degenerative arthritis patients to recover their structures, most treatments focus on decreasing pain or improving functions. ${ }^{8,10}$ It is thus necessary to develop a treatment that improves the knee structure of degenerative arthritis patients. Traction therapy is currently being used to treat spine dysfunction and has been shown to relieve joint

Copylight (C)2019 The Korean Society of Physical Therapy

This is an Open Access article distribute under the terms of the Creative Commons Attribution Non-commercial License (Http:// creativecommons.org/license/by-nc/4.o.) which permits unrestricted non-commercial use, distribution, and reproduction in any medium, provided the original work is properly cited. 
compression and relax muscle. ${ }^{11,12}$ A recent study demonstrated that mechanical joint traction of the knee through the use of an external fixing device on patients with degenerative arthritis showed promising results. ${ }^{13}$ The treatment increased joint space and cartilage thickness, decreased lost brain areas, and improved overall function. ${ }^{13,14}$ Traction therapy with external fixation has also been shown to be effective in reducing pain in patients with degenerative arthritis by maintaining joint space. ${ }^{13}$ Furthermore, this therapy increases joint space, maintains expansion, increases cartilage thickness, reduces bone loss, and improves knee function in patients with degenerative arthritis. ${ }^{14}$ Other recent studies have also shown that knee joint traction therapy may be useful for improving pain and function and reducing depression in patients with degenerative arthritis. ${ }^{14,15}$ Although weight loss, lifestyle change, and drug treatment are ways of physical improvement for degenerative arthritis patients, they are used to delay the progress of disease. ${ }^{16,17}$ However, knee joint traction has shown positive effects in improving the pain, range of joint motion, and the quality of life..$^{14,18}$

Knee joint traction is helpful in improving the pain and functions of degenerative arthritis patients and will become a treatment method to improve the joint structure. However, there are very few studies on knee joint traction in Korea. However, the traction of the joints using external fixation devices is difficult to apply to a large number of people because it requires surgery and causes the inconvenience of wearing the device during daily life. Therefore, this study investigated the impact of knee joint traction therapy on pain, physical function, and depression in patients with degenerative arthritis.

\section{METHODS}

\section{Subjects}

This study included 30 patients who had been diagnosed by their at-

Table 1. General characteristics of subjects

\begin{tabular}{lccc}
\hline Variables & EG $(n=15)$ & CG $(n=15)$ & $p$ \\
\hline Gender (male/female) & $7 / 8$ & $8 / 7$ & \\
Age $(\mathrm{yr})$ & $67.53 \pm 4.13$ & $65.40 \pm 4.88$ & 0.207 \\
Height $(\mathrm{cm})$ & $162.20 \pm 6.20$ & $163.80 \pm 6.57$ & 0.498 \\
Weight $(\mathrm{kg})$ & $59.86 \pm 6.15$ & $60.13 \pm 5.84$ & 0.904 \\
Duration & $12.06 \pm 2.01$ & $13.06 \pm 2.21$ & 0.207 \\
K-L grade (\%) & $2.26 \pm 0.45$ & $2.66 \pm 0.61$ & 0.053 \\
\hline
\end{tabular}

Values are presented as mean \pm standard deviation.

EG: experimental group, CG: control group, K-L grade: Kellgren-Lawrence grade. tending doctors with knee degenerative arthritis based on clinical findings and images taken using X-ray equipment. These subjects were selected from patients who had either been hospitalized at $\mathrm{S}$ Hospital or who had visited the hospital as outpatients. This was a randomized clinical trial. The 30 patients with degenerative arthritis were randomly assigned to one of two groups: the experimental group of patients who underwent knee joint traction therapy or the control group of patients who underwent general physical therapy (15 patients per group). The experiment was conducted without anyone dropping in the middle. The inclusion criteria were: age over 60 years, Kellgren-Lawrence $(\mathrm{K} / \mathrm{L})$ grade $>2$, and not currently exercising. Subjects who had received surgery on the knee joint were excluded. A patient was removed from the experiment if he was receiving drug treatment, had a ligament damage, infection, central nervous disorder, and cognitive disorder. Informed consent was voluntarily obtained from all the subjects before participation in the study, and this study was approved by the institutional human ethics committees, and written informed consent was obtained from all subjects, before their participation. The study also included a signed consent form, according to the ethical standards of the Declaration of Helsinki. The general characteristics of the participants are presented in Table 1.

\section{Interventions}

The experimental and control groups received general physical therapy, which was carried out in three ways and included 20 minutes of superficial heat therapy, 5 minutes of deep heat therapy, and 20 minutes of electric therapy five times a week for 4 weeks. In addi-

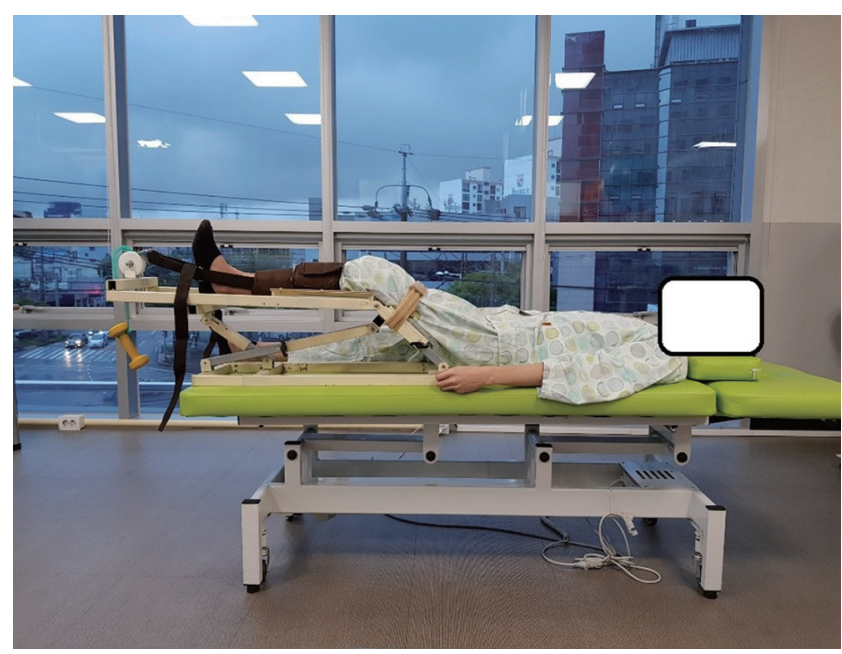

Figure 1. Knee joint traction therapy. 
tion, the experimental group received a knee joint traction workout for 20 minutes a day, five times a week, for 4 weeks. The participants were asked to bend their hip and knee joints at 60 degrees in the supine position. The tibia and thigh were secured with a strap, and continuous knee joint traction treatment was applied to tow the tibia in the cephalocaudal direction. The force that was applied by the traction was approximately equal to $6 \%$ of the participant's weight, ${ }^{19,20}$ and the traction continued for a 20 minute a stretch (Figure 1).

\section{Experimental methods}

Pain was measured using the visual analogue scale (VAS), which evaluates the intensity of subjective pain. The patient scores their pain on a scale of $0-10(0=$ no pain, $10=$ the most severe pain $)$.

Physical function was measured using the Western ontario and McMaster universities osteoarthritis (WOMAC) scale, which measures specific diseases, personal health management, and physical conditions. It is a clinically important functional evaluation tool for assessing pain, stiffening, and physical function in patients with osteoarthritis and coxarthritis. Physical function can be measured by asking the patient to complete a self-reported, and the index consists of 24 categories (5 categories for pain, 2 categories for stiffening, and 17 categories for physical function). All questions are scored on a scale of $0-4$ $(0=$ none, $1=$ mild, $2=$ moderate, $3=$ severe, $4=$ very severe $)$, where higher scores indicate worse pain, stiffness, and functional limitations.

Depression was measured using the Beck depression inventory (BDI), which is a four-point measurement method that includes 21 questions. Its total scores range from 0 to 63,9 points, $10-15$ points, 16-23 points, and 24-63 points equate with no depression, mild depression, depression, and serious depression, respectively. The VAS, WOMAC score, and BDI score were recorded before and after the 4-week treatment.

\section{Statistical analysis}

Collected data were analyzed using SPSS 19.0 (SPSS Inc., Chicago, IL, USA). Descriptive statistics were used to compare the general characteristics of the participants. All data were verified for normality using the Shapiro-Wilks verification test, and the paired t-test was used to compare values before and after the experiment. An independent t-test was also conducted to compare intergroup differences among the changes that occurred after the experiment. The statistical significance level was set at $\alpha=0.05$.

\section{RESULTS}

\section{VAS}

The experimental group exhibited a significant difference as its VAS

Table 2. Comparison of pre-post VAS, WOMAC and BDI between experimental and control group

\begin{tabular}{|c|c|c|c|c|c|}
\hline Variables & & EG & CG & $\mathrm{t}$ & $p$ \\
\hline \multirow[t]{5}{*}{ VAS (score) } & Pre & $7.13 \pm 0.91$ & $6.06 \pm 0.88$ & & \\
\hline & Post & $2.40 \pm 0.91$ & $5.06 \pm 0.79$ & & \\
\hline & Difference & $-4.73 \pm 0.96$ & $-1.00 \pm 1.06$ & -10.058 & $0.000^{*}$ \\
\hline & $\mathrm{t}$ & 19.073 & 3.623 & & \\
\hline & $\mathrm{p}$ & $0.000^{*}$ & $0.003^{*}$ & & \\
\hline \multirow[t]{5}{*}{ WOMAC (score) } & Pre & $47.20 \pm 1.65$ & $44.13 \pm 2.29$ & & \\
\hline & Post & $25.33 \pm 2.38$ & $35.26 \pm 2.76$ & & \\
\hline & Difference & $-21.86 \pm 3.29$ & $-8.86 \pm 3.77$ & -10.049 & $0.000^{*}$ \\
\hline & t & 25.725 & 9.092 & & \\
\hline & $\mathrm{p}$ & $0.000^{*}$ & $0.000^{*}$ & & \\
\hline \multirow[t]{5}{*}{ BDI (score) } & Pre & $22.33 \pm 1.34$ & $19.53 \pm 1.18$ & & \\
\hline & Post & $13.80 \pm 1.61$ & $18.13 \pm 1.59$ & & \\
\hline & Difference & $-8.53 \pm 1.72$ & $-1.40 \pm 1.76$ & -11.19 & $0.000^{*}$ \\
\hline & $\mathrm{t}$ & 19.142 & 3.073 & & \\
\hline & $\mathrm{p}$ & $0.000^{*}$ & $0.008^{*}$ & & \\
\hline
\end{tabular}

Values are presented as mean \pm standard deviation

EG: experimental group, CG: control group, VAS: Visual Analogue Scale, WOMAC: Western Ontario and Macmaster Universities Arthritis Index, BDI: Beck Depression Inventory.

${ }^{*} \mathrm{p}<0.05$. 
score, which decreased from $7.13 \pm 0.91$ before treatment to $2.40 \pm$ 0.91 after treatment $(\mathrm{p}<0.05)($ Table 2$)$. The control group exhibited a significant difference as its VAS score, which decreased from 6.06 \pm 0.88 before treatment to $5.06 \pm 0.79$ after treatment $(\mathrm{p}<0.05)$ (Table 2). In comparison between the two groups, the experimental group in which knee joint traction was applied showed more significant change in VAS than the control group $(\mathrm{p}<0.05)$ (Table 2).

\section{WOMAC}

The experimental group exhibited a significant difference as its WOMAC score, which decreased from $47.20 \pm 1.65$ before treatment to $25.33 \pm 2.38$ after treatment $(\mathrm{p}<0.05)$ (Table 2$)$. The control group exhibited a significant difference as its WOMAC score, which decreased from $44.13 \pm 2.29$ before treatment to $35.26 \pm 2.76$ after treatment $(\mathrm{p}<0.05)$ (Table 2). In comparison between the two groups, the experimental group in which knee joint traction was applied showed more significant change in WOMAC than the control group $(\mathrm{p}<0.05)$ (Table 2).

\section{BDI}

The experimental group exhibited a significant difference as its BDI score, which decreased from $22.33 \pm 1.34$ before treatment to 13.80 \pm 1.61 after treatment $(\mathrm{p}<0.05)$ (Table 2). The control group exhibited a significant difference as its BDI score, which decreased from $19.53 \pm 1.18$ before treatment to $18.13 \pm 1.59$ after treatment $(\mathrm{p}<0.05)$ (Table 2). In comparison between the two groups, the experimental group in which knee joint traction was applied showed more significant change in BDI than the control group $(\mathrm{p}<0.05)$ (Table 2).

\section{DISCUSSION}

This study investigated the impact of knee joint traction therapy on pain, physical function, and depression in patients with degenerative arthritis. According to our results, a decrease in pain was observed in this study after the application of knee joint traction therapy. In the study by Intema et al. ${ }^{13}$ the external surgical fixing device that was used for traction was reported to have positive effects on decreasing pain. Furthermore, Lee and Lee ${ }^{21}$ reported that mechanical traction therapy had a positive impact on pain relief in patients with degenerative gonarthritis. These investigators found that knee joint traction treatment significantly decreased pain, in agreement with the results of our study. Knee joint traction therapy results in muscle relaxation, stimulation of dynamic muscle contractions, and inhibition of protective muscle reflections, all of which are instrumental in decreasing pain. ${ }^{13}$ Moreover, the increase in knee joint space due to continuous knee joint traction also helps reduce pain. ${ }^{14}$

In this study, the application of knee joint traction therapy on patients with degenerative arthritis resulted in improved physical function. Lee et al. ${ }^{22}$ and Alpayci et al. ${ }^{14}$ reported a decrease in WOMAC scores after applying knee joint traction therapy on patients with degenerative arthritis. Khademi-Kalantari et al. ${ }^{18}$ also demonstrated improvements in physical function and gait ability in patients with degenerative arthritis after applying knee joint traction treatment. During traction therapy, which is generally performed on the spine, it is difficult to generate traction by separating only one joint, due to its structural characteristics. ${ }^{23}$ However, traction therapy on the knee joint has the advantage of focusing traction on one segment and can be used to improve pain, tissue structure, and physical function by reducing joint contact and pressure on joint cartilage during movement and securing joint space. When the knee joint traction was applied in van Valburg et al. ${ }^{15}$ research, it was effective for increasing the joint space and cartilage space, decreasing the amount of brain part lost, and the functions. Increased joint space by traction improves symptoms through neurophysiological effects which control harmful information from the tissue, and it is reported that pain decreases by relaxing muscles. ${ }^{24}$

In patients with knee degenerative arthritis, depression and pain were correlated, and pain was shown to have a negative influence on patients. In this study, both depression and knee pain decreased upon the application of traction therapy, which normalized the movements of the lower limbs during daily life activities. Knee joint traction therapy has also previously been found to significantly decrease depression, in agreement with this study's result. Improvements in quality of life will also help with depression, and Lee et al. ${ }^{21}$ identified improvements in the quality of life of patients with degenerative arthritis after traction therapy. Based on these results, traction therapy is thought to improve pain, physical function, and depression in patients with degenerative arthritis, all of which were confirmed to improve after therapy in the patients with degenerative arthritis in this study. This study was conducted to examine the effect of knee joint traction on degenerative arthritis in improving their pain, physical functions, and depression. Given that most trac- 
tions are focused on spine, little research has been carried out in Korea. Hence, this research has huge significance as it has applied the knee joint traction therapy on degenerative arthritis patients.

There are a few limitations to this study. First, it is difficult to generalize our results because of the small number of participants. Furthermore, we could not conduct a follow-up study to investigate the long-term the effects of continuous knee joint traction after treatment termination. To generalize the results of our study, it will be necessary to conduct a long-term follow-up study using a larger cohort of patients with knee joint arthritis in the future.

\section{REFERENCES}

1. Altman RD, Moskowitz R. Intraarticular sodium hyaluronate (Hyalgan) in the treatment of patients with osteoarthritis of the knee: a randomized clinical trial. Hyalgan Study Group. J Rheumatol. 1998;25(11):2203-12.

2. Migliore A, Procopio S. Effectiveness and utility of hyaluronic acid in osteoarthritis. Clin Cases Miner Bone Metab. 2015;12(1):31-3.

3. Park SJ, Lee JH. Effect of joint mobilization and kinesio taping on pain, range of motion, and knee function in patients with knee osteoarthritis. J Kor Phys Ther. 2016;28(5):279-85.

4. Felson DT, Anderson JJ. Across-study evaluation of association between steroid dose and bolus steroids and avascular necrosis of bone. Lancet. 1987;1(8538):902-6.

5. Lee JH, Cho HS, Song IY. Effectiveness of shortwave therapy in management of knee osteoarthritis: A systematic review and meta-analysis of randomized controlled trials. J Kor Phys Ther. 2014;26(5):331-43.

6. McKay C, Prapavessis H, Doherty T. The effect of a prehabilitation exercise program on quadriceps strength for patients undergoing total knee arthroplasty: a randomized controlled pilot study. PM R. 2012;4(9):64756.

7. Turner JA, Ersek M, Kemp C. Self-efficacy for managing pain is associated with disability, depression, and pain coping among retirement community residents with chronic pain. J Pain. 2005;6(7):471-9.

8. Hochberg MC, Altman RD, April KT et al. American College of Rheumatology 2012 recommendations for the use of nonpharmacologic and pharmacologic therapies in osteoarthritis of the hand, hip, and knee. Arthritis Care Res (Hoboken). 2012;64(4):465-74.

9. Ylldırım MA, Uçar D, Öneș K. Comparison of therapeutic duration of therapeutic ultrasound in patients with knee osteoarthritis. J Phys Ther Sci. 2015;27(12):3667-70.

10. Zhang W, Moskowitz RW, Nuki G et al. OARSI recommendations for the management of hip and knee osteoarthritis, Part II: OARSI evidence-based, expert consensus guidelines. Osteoarthritis Cartilage. 2008;16(2):137-62.

11. Krause M, Refshauge KM, Dessen M et al. Lumbar spine traction: evaluation of effects and recommended application for treatment. Man Ther. 2000;5(2):72-81.

12. Zusman M. The absolute visual analogue scale (AVAS) as a measure of pain intensity. Aust J Physiother. 1986;32(4):244-6.

13. Intema F, Van Roermund PM, Marijnissen AC et al. Tissue structure modification in knee osteoarthritis by use of joint distraction: an open 1-year pilot study. Ann Rheum Dis. 2011;70(8):1441-6.

14. Alpayci M, Ozkan Y, Yazmalar L et al. A randomized controlled trial on the efficacy of intermittent and continuous traction for patients with knee osteoarthritis. Clin Rehabil. 2013;27(4):347-54.

15. van Valburg AA, van Roermund PM, Marijnissen AC et al. Joint distraction in treatment of osteoarthritis: a two-year follow-up of the ankle. Osteoarthritis Cartilage. 1999;7(5):474-9.

16. Bjordal JM, Johnson MI, Lopes-Martins RA. Short-term efficacy of physical interventions in osteoarthritic knee pain. A systematic review and meta-analysis of randomised placebo-controlled trials. BMC Musculoskelet Disord. 2007;8(1):51.

17. van Nguyen J, Marks R. Pulsed electromagnetic fields for treating osteoarthritis. Physiotherapy. 2002;88(8):458-70.

18. Khademi-Kalantari K, Mahmoodi Aghdam S, Akbarzadeh Baghban A et al. Effects of non-surgical joint distraction in the treatment of severe knee osteoarthritis. J Bodyw Mov Ther. 2014;18(4):533-9.

19. de Leva P. Adjustments to Zatsiorsky-Seluyanov's segment inertia parameters. J Biomech. 1996;29(9):1223-30.

20. Plagenhoef S, Evans FG, Abdelnour T. Anatomical data for analyzing human motion Res Q Exerc Sport. 1983;54(2):169-78.

21. Lee DK, Lee NY. Case study of continuous knee joint traction treatment on the pain and quality of life of patients with degenerative gonarthritis. J Phys Ther Sci. 2018;30(6):848-9.

22. Lee NY, Kwon CS, Kim SY. The effect of mechanical traction on pain and physical function in patients with knee osteoarthritis. Phys Ther Korea. 2015;22(3):23-32.

23. Krause M, Refshauge KM, Dessen M et al. Lumbar spine traction: Evaluation of effects and recommended application for treatment. Man Ther. 2000;5(2):72-81.

24. Twomey LT. Sustained lumbar traction. An experimental study of long spine segments. Spine (Phila Pa 1976). 1985;10(2):146-9. 